Trab-679-00 (6 páginas)

\title{
USO DE INFORMAÇÕES OBTIDAS NO TRANSPORTE DE MADEIRA E NO INVENTÁRIO FLORESTAL PARA ESTIMAR O VOLUME EM ESTERÉOS ${ }^{1}$
}

\author{
Valdir Carlos Lima de Andrade ${ }^{2}$, Helio Garcia Leite ${ }^{3}$ e Antonio do Nascimento Gomes ${ }^{4}$
}

\begin{abstract}
RESUMO - O objetivo deste trabalho foi desenvolver uma metodologia para estimação do volume em estéreos sem uma amostragem prévia do empilhamento das toras. Os dados necessários são provenientes do inventário florestal e do transporte da madeira. Foi adotado o "método dos espaços vazios" para ilustrar o emprego das informações supracitadas. Foram utilizados dados de 91 parcelas de inventário pré-corte e de transporte do volume de um plantio de híbrido Eucalyptus grandis x Eucalyptus urophylla, com uma área de 243,1 ha. As equações geradas para estimar o volume em estéreos resultaram em um erro de $-0,76 \%$ ou $-2,34$ st/ha para a equação advinda de deduções do método dos espaços vazios e de $4,54 \%$ ou 11,61 st/ha para a equação gerada com o emprego do modelo de taper de Demaerschalk. Estes resultados evidenciam que a metodologia apresentada gera resultados promissores, permitindo utilizar informações representativas da situação de uso da unidade estéreos.
\end{abstract}

Palavras-chave: Volume transportado, espaço vazio, estéreos e inventário florestal.

\section{INFORMATION OBTAINED FROM TIMBER TRANSPORTATION AND FOREST INVENTORY FOR THE ESTIMATION OF VOLUME IN STERES}

\begin{abstract}
The objective of this work was to develop a methodology to estimate volume in steres (st) without a previous log staking sampling. Data from a forest inventory and timber transportation were used. The "Empty Spaces Method" was adopted to illustrate the application of the information. Data on 91 plots of a pre-cut and transport inventory of a Eucalyptus grandis $x$ Eucalyptus urophylla hybride planting volume, with na area of $243.1 \mathrm{ha}$, were utilized. The equations generated to estimate the volume in steres resulted in an error of $-0.76 \%$ or -2.34 st/ha, for the equation generated from the "empty spaces method" deductions and of $4.54 \%$ or 11.61 st/ha, for the equation generated from application the Demaerschalk taper model. These results indicate that this methodology generates promising results, allowing to apply representative information on the use of the steres unit.
\end{abstract}

Key words: Transported volume, empty space, steres and forest inventory.

\section{INTRODUÇÃO}

Um dos métodos usuais de determinação do volume na unidade estéreos consiste no uso do fator de empilhamento, definido pela razão entre o volume em estéreos e o volume sólido da pilha. Outro método, apresentado em Andrade \& Leite (1998), consiste em utilizar os espaços vazios existentes entre as toras empilhadas para obter um fator de correção, definido como a razão entre a área de espaços vazios e a área de espaços ocupados com madeira..

1 Recebido para publicação em 16.11.2000.

Aceito para publicação em 19.2.2003.

2 Engenheiro Florestal, M.S., Rua Octávio Cerqueira, 31, Vila Oliveira, 18200-000 Itapetininga-SP, <vclandrade@mailcity.com>.

${ }^{3}$ Dep. de Engenharia Florestal da Universidade Federal de Viçosa - UFV, 36571-000 Viçosa-MG, <hgleite@mail.ufv.br>.

${ }^{4}$ Engenheiro Florestal, M.S., <nasci.gomes@ig.com.br> 
Para estimação do volume em estéreos, independentemente do método adotado, utilizam-se informações coletadas de amostras de toras empilhadas, em geral, por classe de diâmetro, advindas de árvores abatidas e seccionadas com comprimento desejado (Rezende, 1988; Silva, 1991; Paula Neto \& Rezende, 1992; Paula Neto et al., 1993; Andrade, 1996).

As toras transportadas até um centro consumidor têm diâmetros variados, isto é, não são separadas por classe de diâmetro. Também, essas toras, possuem um teor de umidade menor que o da árvore em pé, ou aquele verificado após o abate das árvores. Além disso, quando a madeira é transportada com casca, parte dessa casca pode ser perdida durante a viagem. Essas situações são diferentes daquelas que geraram as equações para estimar o fator de empilhamento e, ou, o fator de correção e, por conseguinte, para estimar o volume em estéreos.

Com base nas argumentações citadas, desenvolveuse este trabalho, objetivando utilizar a informação de volume de madeira já transportada na determinação do volume em estéreos em inventários florestais, por acreditar ser uma situação mais representativa desta variável, independentemente do método empregado na colheita ou do tipo de transporte das toras.

\section{MATERIAL E MÉTODOS}

Foram utilizados, em um estudo de caso, dados de 91 parcelas circulares com $471,44 \mathrm{~m}^{2}$ cada, oriundas do banco de dados do Inventário Florestal Pré-Corte da empresa Copener Florestal Ltda., sediada no município de Alagoinhas-BA. As 91 parcelas foram distribuídas aleatoriamente em 11 talhões, que perfazem uma população de 243,1 ha do híbrido Eucalyptus grandis W. Hill ex Maiden x Eucalyptus urophylla S. T. Blake, cujo material se refere a uma floresta em regime de alto fuste, com 6 anos de idade e em primeira rotação.

As informações utilizadas foram o volume sólido estimado em cada talhão e aquelas obtidas na cubagem rigorosa realizada durante a condução do inventário florestal. Foram consideradas, também, as informações do volume transportado de madeira colhida na área. Estas informações foram separadas em dois lotes de dados. Um lote, contendo cinco talhões, foi utilizado para gerar equações, e o segundo, contendo os outros seis talhões, foi utilizado na avaliação das estimativas obtidas do volume em estéreos de cada talhão utilizado. Tais volumes, $\mathrm{em} \mathrm{m}^{3} \mathrm{e}$ em estéreos, referem-se ao uso comercial até o diâmetro de $6 \mathrm{~cm}$ sem casca (Quadro 1).

Quadro 1 - Dados utilizados no desenvolvimento e na aplicação das equações para estimar o volume em estéreos até $6 \mathrm{~cm}$ de diâmetro sem casca

Table 1 - Data used in the development and application of the equations for the estimation of the volume in steres up to 6 cm of diameter inside bark

\begin{tabular}{|c|c|c|c|}
\hline Talhão* & Área (ha) & Volume transportado $s c$ & No de Parcelas \\
\hline 03 & 90,46 & $25.503,7$ & 30 \\
04 & 3,64 & 650,4 & 5 \\
06 & 8,73 & $2.502,9$ & 5 \\
10 & 7,50 & $2.584,0$ & 5 \\
11 & 11,00 & $3.588,5$ & 5 \\
\hline Total & 121,3 & $34.829,5$ & 50 \\
\hline \multicolumn{2}{|c|}{ Dados utilizados para gerar equacos (Data used in the development of the equations) } \\
\hline 01 & 26,00 & $5.530,9$ & 9 \\
05 & 20,95 & $4.556,0$ & 7 \\
07 & 21,32 & $5.885,4$ & 7 \\
08 & 17,59 & $5.616,7$ & 6 \\
\hline Total & 22,00 & $7.667,5$ & 7 \\
\hline Total Geral & 13,92 & $4.924,8$ & 5 \\
\hline
\end{tabular}

* Foi considerado como pilha de madeira, pois o volume em estéreos, transportado por caminhões, foi totalizado por talhão.

R. Árvore, Viçosa-MG, v.27, n.2, p.171-176, 2003 


\subsection{Obtenção das Equações para Estimar o Volume em Estéreos}

As informações de volume $\mathrm{em}^{3} \mathrm{e}$ em estéreos dos cinco talhões foram aplicadas no método dos espaços vazios, que foi apresentado em Andrade \& Leite (1998). Este método segue o seguinte princípio: " $O$ volume $n a$ unidade estéreos, de qualquer ponto no tronco de uma árvore, pode ser estimado por qualquer um dos métodos existentes, seja de taper, de razão volumétrica, de múltiplos volumes ou de único volume se acrescentado, aos diâmetros advindos de uma cubagem rigorosa, os espaços vazios ocasionados pelo formato circular das toras empilhadas".

Para se obter equações de volume em estéreos empregando o fator de correção, neste trabalho, aplicouse:

$$
F c_{j}=\frac{g e_{j}}{g m_{j}}
$$

sendo $g e_{j}=\frac{V s t_{j}-V m_{j}^{3}}{\bar{h}_{j}}$ e $g m_{j}=\frac{1}{n_{j}} \cdot\left[\sum_{j=1}^{n_{j}}\left(\frac{V m_{i j}^{3}}{h_{i j}}\right)\right]$,

em que $F c_{j}$ é o fator de correção obtido no j-ésimo talhão; $g e_{j}$ e $g m_{j}$ são a área contendo espaços vazios e a área contendo somente madeira no j-ésimo talhão, $\mathrm{em} \mathrm{m}^{2} / \mathrm{ha}$;

$V s t_{j}$ e $V m_{j}^{3}$ são os volumes de madeira existentes no j-ésimo talhão, em estéreos/ha e em $\mathrm{m}^{3} / \mathrm{ha} ; n_{j}$ é o número de árvores amostradas no j-ésimo talhão; $h_{i j}$ é a altura referente ao diâmetro comercial da i-ésima árvore amostrada no j-ésimo talhão, em metros; $V m_{i j}^{3}$ é o volume comercial da i-ésima árvore amostrada no j-ésimo talhão, em m ${ }^{3} ; \bar{h}_{j}$ é a média aritmética de $h_{i j}$ no j-ésimo talhão.

O volume comercial transportado no j-ésimo talhão, para efeito do desenvolvimento e aplicação do método, foi considerado como sendo o volume real, e os dados da cubagem rigorosa que totalizaram 188 árvores-amostra foram usados para ajuste do modelo de Demaerschalk (1973), visando obter as equações para empregar na estimação do volume sólido das parcelas do inventário florestal no j-ésimo talhão. Assim, utilizando a equação 1, calculou-se o fator de correção de cada um dos cinco talhões utilizados. Em seguida, utilizando os procedimentos apresentados em Andrade (1996) e em Andrade \& Leite (1998), obteve-se a expressão:

$$
d m e=d \cdot \sqrt{F c+1}
$$

em que $d$ e $d m e$ são o diâmetro contendo somente madeira e o diâmetro contendo madeira acrescida de espaços vazios, respectivamente.

Aplicando a expressão equação 2 nos dados das 188 árvores cubadas, foram gerados os valores de $d m e$ que foram empregados no ajuste do modelo de Demaerschalk (1973). A integral da equação obtida por este ajuste resultou em uma equação volumétrica para ser empregada na estimação do volume em estéreos de multiprodutos, sem o uso de fator de empilhamento.

Também, utilizando a equação 1 , obteve-se uma outra equação para estimar o volume em estéreos sem o uso do fator de empilhamento, ou seja, utilizando as expressões de $g e_{j}$ e $g m_{j}$ e o artifício de que as variáveis $h_{i j}$ e $V m_{i j}^{3}$ são iguais a $\bar{h}_{j}$ e $V m_{j}^{3}$ deduziu-se que:

$$
F c_{j}=\frac{V s t_{j}-V m_{j}^{3}}{\bar{h}_{j}} \cdot \frac{\bar{h}_{j}}{V m_{j}^{3}} \Leftrightarrow F c_{j}=\frac{V s t_{j}}{V m_{j}^{3}}-1 \Leftrightarrow F c_{j}+1=\frac{V s t_{j}}{V m_{j}^{3}},
$$

ou:

$V s t_{j}=V m_{j}^{3} \cdot\left(F c_{j}+1\right)$

\subsection{Análise da Precisão das Estimativas}

Para avaliação das estimativas de volume em estéreos dos seis talhões restantes, foram utilizadas as estatísticas referentes ao erro médio porcentual (EMP), à raiz quadrada do erro médio $(R Q E M)$ e ao Bias, obtidas pelas fórmulas:

$E M P=\frac{1}{6} \cdot \sum_{j=1}^{6}\left[\left(\frac{\hat{Y}_{j}-Y_{j}}{Y_{j}}\right) \cdot 100\right]$,

R. Árvore, Viçosa-MG, v.27, n.2, p.171-176, 2003 
$R Q E M=\sqrt{\frac{\sum_{j=1}^{6}\left(\hat{Y}_{j}-Y_{j}\right)^{2}}{6}} \mathrm{e}$

Bias $=\frac{1}{6} \cdot\left(\sum_{j=1}^{6} \hat{Y}_{j}-\sum_{j=1}^{6} Y_{j}\right)$,

em que $\hat{Y}_{j}$ e $Y=$ respectivamente, volume estimado e observado no j-ésimo talhão, em estéreos/ha.

Procedeu-se, também, à validação das equações por meio do teste F (Graybill, 1976), considerando as recomendações de Guimarães (1994).

\section{RESULTADOS E DISCUSSÃO}

\subsection{Estudo de Caso}

A equação obtida pelo ajuste do modelo de Demaerschalk, utilizando os diâmetros cubados em centímetros sem casca, foi

$$
\left(\frac{\hat{d}}{d a p}\right)=10^{0,052599} \cdot \operatorname{dap}^{0,923084} \cdot(H t-h)^{0,751113} \cdot H t^{-0,716868}
$$

com um coeficiente de correlação linear $\left(r_{\hat{Y} Y}\right)$ de 0,972 . A partir de transformações na equação 4 , obteve-se que:

$$
\begin{gathered}
\hat{V m^{3}}=\frac{\pi}{40000} \cdot 10^{0,105198} \cdot d a p^{1,846168} \cdot H t^{-1,433736} \\
\cdot\left[\frac{(H t-0,1)^{2,502226}-(H t-\hat{h})^{2,502226}}{2,502226}\right] \\
\hat{h}=H t-\left(\frac{d c]}{10^{0,052599} \cdot d a p^{0,923084} \cdot H t^{-0,716868}}\right)^{\frac{1}{0,751113}}
\end{gathered}
$$

em que $d c$ é o diâmetro comercial desejado, que, neste trabalho, utilizou-se $6 \mathrm{~cm} \mathrm{sc}$.

Conforme reportado em 2.1., as equações 5 e 6 foram utilizadas para estimar o volume sólido sem casca por árvore $\left(\hat{\mathrm{Vm}^{3}}\right)$. Nesse mesmo item, obteve-se o fator de correção que, após o ajuste do modelo estatístico $\operatorname{Ln}\left(F c_{j}\right)=\beta_{0} \cdot \overline{H t}_{j}{ }_{j}^{\left(\beta_{1}\right)} \cdot \bar{D}_{j}{ }^{\left(\beta_{2}\right)} \cdot \varepsilon$, gerou a equação:

$\hat{F c}_{j}=\operatorname{EXP}\left(-8327557 \cdot \overline{H t}_{j}^{(-0,10986)} \cdot \bar{D}_{j}^{(-6,10072)}\right)$,

$\operatorname{com} r_{\hat{Y} Y}=0,867$

em que $L n=$ logaritmo natural; $E X P=$ inverso de $L n$;

$\hat{F C}_{j}=$ fator de correção estimado para o j-ésimo talhão;

$\overline{H t}_{j}$ e $\bar{D}_{j}=$ médias aritméticas no j-ésimo talhão da altura total e do dap; $\beta_{i}=$ parâmetros da equação a estimar; e $\varepsilon=$ erro aleatório.

Substituindo a equação 7 na equação 3 e considerando o volume sólido estimado pela equação 5 , que foi totalizado por hectare e talhão, obteve-se:

$$
\hat{V s t} / h a_{j}=\hat{V m^{3}} / h a_{j} \cdot\left[\operatorname{EXP}\left(-8327557 \cdot \overline{H t}_{j}^{(-0,10986)} \cdot \bar{D}_{j}^{(-6,10072)}\right)+1\right]
$$

Esta expressão foi utilizada para estimar o volume na unidade estéreos por hectare no j-ésimo talhão.

Substituindo a equação 7 na equação 2 e considerando as variáveis $\overline{H t}_{j}$ e $\bar{D}_{j}$, respectivamente, como $H t$ e dap, obteve-se a expressão:

$$
\hat{d m e}=d \cdot \sqrt{\operatorname{EXP}\left(-8327557 \cdot H t^{-0,10986} \cdot d a p^{-6,10072}\right)+1}
$$

A expressão anterior foi aplicada nas 188 árvoresamostra cubadas, tendo sido obtidas as estimativas dos diâmetros contendo madeira acrescida dos espaços vazios ocasionados pelo formato circular das toras empilhadas nos caminhões durante o transporte. Em seguida, utilizando valores de dme estimados, ajustou-se 
novamente o modelo de Demaerschalk, resultando na equação:

$$
\left(\frac{\hat{d m e}}{d a p}\right)=10^{-0,836071} \cdot d a p^{0,565549} \cdot(H t-h)^{0,731046} \cdot H t^{0,352939}
$$

$\operatorname{com} r_{\hat{Y} Y}=0,835$

Por transformação matemática dessa equação de taper obteve-se a expressão

$$
\begin{aligned}
\hat{V s t} & =\frac{\pi}{40000} \cdot 10^{-1,672142} \cdot d a p^{1,131098} . H t^{0,705878} \\
& \cdot\left[\frac{(H t-0,1)^{2,462092}-(H t-\hat{h})^{2,462092}}{2,462092}\right]
\end{aligned}
$$

empregada para estimar o volume em estéreos de árvores individuais em cada talhão.

\subsection{Análise das Estimativas do Volume em Estéreos}

As equações 8 e 9 foram aplicadas em todas as parcelas dos seis talhões selecionados especificamente para esta atividade. Em seguida, foram obtidas as estatísticas $E M P, R Q E M$ e Bias. Estimaram-se ainda os erros mínimo (Emin) e máximo (Emax) porcentuais das estimativas geradas. Estas estatísticas foram utilizadas para classificar as alternativas de estimação do volume em estéreos (equações 8 e 9), conforme Quadro 2.

Analisando os resultados do Quadro 2, nota-se que as estatísticas referentes à equação 8 apresentaram os menores valores, de forma expressiva principalmente para o Emax, EMP e Bias.

Pelo teste F Graybill não foram constatadas diferenças significativas (Quadro 3), sendo, então, ambas alternativas incluídas no caso 1 de estimação, conforme Guimarães (1994), que parece ser uma situação ideal de predizer volume em estéreos.

Ao serem considerados os 121,8 ha dos seis talhões e os resultados do Bias (-2,34 st/ha e 11,61 st/ha), obtevese uma subestimação de 285,012 estéreos pelo uso da equação 8 contra uma superestimação de 1.414,098 estéreos pelo uso da equação 9, o que demonstra a superioridade da equação 8 para estimar o volume em estéreos. Se for necessário contemplar diferentes usos comerciais, a equação 9 pode ser utilizada desde que seja aceita a superestimação de $1.414,098$ estéreos para a população de 121,8 ha.

Cabe lembrar que informações de inventários florestais anteriores, em outros projetos, juntamente com dados

Quadro 2 - Valores de Emin., Emax., EMP, RQEM e Bias obtidos pela aplicação das equações 8 e 9 nas parcelas dos seis talhões utilizados para classificar as alternativas de estimação do volume em estéreos

Table 2 - Emin., Emax., EMP, RQEM and Bias values obtained from applying equations 8 and 9 to the plots of the six stand used to classify the alternatives of estimating volumes in steres

\begin{tabular}{|c|c|c|c|c|c|}
\hline Equação & Emin (\%) & Emax (\%) & EMP (\%) & RQEM (st/ha) & Bias (st/ha) \\
\hline$e q .8$ & $-0,21$ & 8,68 & $-0,76$ & 15,36 & $-2,34$ \\
$e q .9$ & 0,23 & 12,65 & 4,54 & 17,26 & 11,61 \\
\hline
\end{tabular}

Quadro 3 - Valores obtidos pela aplicação do teste F Graybill nas estimativas em estéreos utilizando as equações 8 e 9 Table 3 -Values obtained from the F Graybill test application in steres estimations using equations 8 and 9

\begin{tabular}{|c|c|c|c|c|c|}
\hline Equação & $\hat{R}^{2}$ & QMRes & CV\% & F & $r_{\hat{\wedge}}$ \\
\hline eq.8 & 0,9144 & 338,2012 & 6,39 & $0,09^{\mathrm{ns}}$ & 0,965 \\
eq.9 & 0,9402 & 236,1120 & 5,34 & $1,79^{\mathrm{ns}}$ & 0,976 \\
\hline
\end{tabular}

Resultados do ajuste da equação: $V s t / h a=\beta_{0}+\beta_{1} \cdot V s t / h a+\varepsilon$. 
de cubagens realizadas nos mesmos e informações sobre o volume transportado, em estéreos, são utilizadas para obter equações a serem usadas em inventários atuais. Ou seja, a metodologia pode ser utilizada com dados anteriores à sua aplicação.

\section{CONCLUSÃO}

Com base nos resultados obtidos, pôde-se concluir que o uso do volume de madeira empilhada, advinda de medições diretas realizadas no veículo do transporte, em situações passadas, juntamente com dados de inventários passados, permite estimar o volume em estéreos em inventários florestais atuais.

\section{REFERÊNCIAS BIBLIOGRÁFICAS}

ANDRADE, V. C. L. Estimativa do volume de multiprodutos, em metro esteres, para árvores de Eucalyptus grandis. 1996. 55 f. Monografia (Graduação em Engenharia Florestal) - Universidade Federal de Viçosa, Viçosa-MG, 1996.

ANDRADE, V. C. L.; LEITE, H. G. Um método para quantificar multiprodutos de árvores individuais na unidade esteres. Revista Árvore, v. 22, n. 3, p. 299-306, 1998.

DEMAERSCHALK, J. P. Integrated systems for the estimation of tree taper and volume. Canadian Journal of Forest Research, v. 3, n. 1, p. 90-94, 1973.
GUIMARÃES, D. P. Desenvolvimento de um modelo de distribuição diamétrica de passo invariante para prognose e projeção da estrutura de povoamentos de eucalipto. 1994. 160 f. Tese (Doutorado em Ciência Florestal) - Universidade Federal de Viçosa, Viçosa-MG, 1994.

GRAYBILL, F. A. Theory and application of the linear model. Massachusetts, Duxbury Press, 1976. 704 p.

PAULA NETO, F.; REZENDE, A. V. Equações de fatores de empilhamento e tabelas de volumes em metros estéreos para árvores individuais. Revista Árvore, v. 16, n. 1, p. 7287, 1992.

PAULA NETO, F. et al. Análise do comportamento dos fatores de empilhamento para Eucalyptus grandis. Revista Árvore, v. 17, n. 1, p. 45-59, 1993.

REZENDE, A. V. Análise dos métodos de estimação do volume sólido da madeira e do comportamento do fator de empilhamento para Eucalyptus grandis. 1988. $131 \mathrm{f}$. Dissertação (Mestrado em Ciência Florestal) - Universidade Federal de Viçosa, Viçosa-MG, 1988.

SILVA, J. A. Estimativas de volume do tronco e da copa, Densidade básica, Fator de empilhamento para madeira da copa e rendimento de madeira serrada de eucalipto. 1991. 90 f. Dissertação (Mestrado em Ciência Florestal) Universidade Federal de Viçosa, Viçosa-MG, 1991. 